\title{
Conservación
}

\section{Registros notables y listado avifaunístico en un área de la Reserva de la Biosfera Sierra Gorda de Guanajuato, México}

\author{
Noteworthy records and avifauna in an area of the Sierra Gorda Biosphere Reserve, \\ Guanajuato, Mexico
}

\author{
Jorge E. Ramírez-Albores ${ }^{\mathrm{a}, *}$, Alejandro Gordillo-Martínez ${ }^{\mathrm{b}}$ y Adolfo G. Navarro-Sigüenza ${ }^{\mathrm{b}}$ \\ ${ }^{a}$ Instituto Potosino de Investigación Científica y Tecnológica, A. C., Camino a la Presa San José 2055, Col. Lomas 4. ${ }^{a}$ sección, \\ 78216, San Luis Potosí, San Luis, Potosí, México \\ ${ }^{\mathrm{b}}$ Museo de Zoología, Facultad de Ciencias, Universidad Nacional Autónoma de México, Apartado Postal 70-399, 04510, México, D. F., México
}

Recibido el 10 de diciembre de 2014; aceptado el 8 de junio de 2015

Disponible en Internet el 11 de noviembre de 2015

\begin{abstract}
Resumen
Documentamos nuevos registros y ampliaciones en el área de distribución de 7 especies de aves en la Reserva de la Biosfera de Sierra Gorda de Guanajuato, México. Cinco de estas especies (Trogon mexicanus, Ara militaris, Cyanocorax yncas, Euphonia elegantissima y Spinus notatus) amplían su distribución conocida y 2 (Buteogallus anthracinus y Myiozetetes similis) no estaban documentadas para la entidad, aunado con el registro de otras 98 especies de aves observadas en esta región. Nuestros registros denotan la importancia de esta región a nivel estatal como un área importante de riqueza de especies de aves y como zona de contacto de aves de afinidad tropical distribuidas en el este de México con las de ambientes secos del Altiplano Mexicano, así como por la presencia de especies indicadoras de buen estado de conservación de los hábitats de bosque. Un mayor esfuerzo de investigación permitirá completar el conocimiento de la avifauna de la Sierra Gorda en Guanajuato y así poder evaluar el estado de conservación real de la comunidad de aves y de las poblaciones que la componen.

Derechos Reservados () 2015 Universidad Nacional Autónoma de México, Instituto de Biología. Este es un artículo de acceso abierto distribuido bajo los términos de la Licencia Creative Commons CC BY-NC-ND 4.0.
\end{abstract}

Palabras clave: Aves; Diversidad; Nuevos registros; Inventario; Sierra Madre Oriental

\section{Abstract}

We report new records and range extensions for 7 bird species in the Biosphere Reserve Sierra Gorda, Guanajuato, Mexico. Five of these species (Trogon mexicanus, Ara militaris, Cyanocorax yncas, Euphonia elegantissima and Spinus notatus) extend their known distribution and 2 (Buteogallus anthracinus and Myiozetetes similis) were not reported for the state, in addition to 98 other bird species observed in this region. Our records indicate the importance of this region at state and national level as an important area for bird species richness and as contact area of tropical bird affinity distribution in eastern Mexico to the dry conditions with the Mexican Plateau, as well as the presence of indicator bird species of good conditions of forest habitats. A greater research effort will allow complete knowledge of the birds in the Sierra Gorda in Guanajuato and be able to assess the real state of conservation of the bird community and populations within it.

All Rights Reserved (C) 2015 Universidad Nacional Autónoma de México, Instituto de Biología. This is an open access item distributed under the Creative Commons CC License BY-NC-ND 4.0.

Keywords: Birds; Diversity; New records; Inventory; Sierra Madre Oriental

\footnotetext{
* Autor para correspondencia.

Correo electrónico: jorgeramirez22@ hotmail.com (J.E. Ramírez-Albores).

La revisión por pares es responsabilidad de la Universidad Nacional Autónoma de México.
}

\section{Introducción}

La avifauna en el estado de Guanajuato, en general, ha sido poco estudiada y existen escasos registros sobre su distribución en esta entidad (Rodríguez-Yáñez, Villalón y Navarro-Sigüenza, 
1994), y este conocimiento es aún más escaso en la zona serrana. Los estudios realizados en el estado son pocos comparado con otras entidades cercanas, como, por ejemplo: Veracruz, Distrito Federal o Jalisco (Navarro-Sigüenza y Benítez, 1993; Navarro-Sigüenza et al., 2014; Rodríguez-Yáñez et al., 1994). Además, los pocos estudios existentes han sido esporádicos, en varios casos asociados a tesis de licenciatura $u$ otras contribuciones no formalmente publicadas que se han limitado a realizar inventarios (e.g., Gómez-Rodríguez, 2011; Moya, 2012; Sánchez-Cordero, 2007), informes ocasionales de registros faunísticos y notas sobre la biología de especies (Bolaños et al., 2012; Donagho, 1965; Moore, 1939), así como estudios ecológicos en ambientes perturbados (Zuria, 2003; Zuria y Gates, 2013) y en ambientes acuáticos (Uriarte-Garzón, 2012; Uriarte-Garzón y Lozoya, 2009). La mayoría de los estudios se han concentrado en la sierra de Santa Rosa y la laguna de Yuriria (Cruz, 1986; Chapa-Vargas, Salgado-Ortiz y Monzalvo-Santos, 2011; Estrada, 1995; González, 2012; Labarthe, 2004). Es notable, sobre todo, que hasta 2004 no existían referencias bibliográficas específicas sobre la diversidad de aves para la pequeña sección de la Sierra Madre Oriental que existe dentro del estado de Guanajuato (Navarro-Sigüenza, Garza-Torres, López de Aquino, Rojas-Soto y Sánchez-González, 2004), donde solamente se detectaron registros de 2 especies (Myioborus pictus y Piranga rubra; Navarro-Sigüenza et al., 2004).

Recientemente Gurrola-Hidalgo, Escalante, López y Sanabria (2012), en la primera síntesis disponible sobre las aves del estado, mencionan que se cuenta con un listado de 366 especies de aves para la entidad, aunque esto no significa que ya se tenga un inventario completo de la avifauna (RojasSoto y Oliveras de Ita, 2005). En este sentido, la necesidad de actualizar la información y generar nuevos estudios ornitológicos toma mayor importancia en la zona de la Sierra Gorda en Guanajuato, un sitio muy poco conocido biológicamente y que es adyacente a otra reserva de la biosfera del mismo nombre en el estado de Querétaro, la cual ha sufrido una intensa pérdida de hábitat y la fragmentación drástica en las últimas décadas (Esparza-Claudio, 2012). En la presente contribución se proporciona información de 7 especies de aves que constituyen registros notables para el estado de Guanajuato, así como un listado de todas las aves observadas durante el estudio.

\section{Materiales y métodos}

La Sierra Gorda en Guanajuato está localizada al noreste de la entidad, cuenta con una superficie de 236,882 ha y comprende parte del municipio de San Luis de la Paz y la totalidad de los municipios de Atarjea, Santa Catarina, Victoria y Xichú $\left(21^{\circ} 41^{\prime}, 2^{\circ} 05^{\prime} \mathrm{N}^{\circ} 99^{\circ} 13^{\prime}, 1^{\circ} 0^{\circ} 13^{\prime}\right.$ O; fig. 1) (Conanp, 2005). Esta región es adyacente a la Reserva de la Biosfera Sierra Gorda en el estado de Querétaro (fig. 1) y forma parte de 2 zonas fisiográficas, la del Altiplano Mexicano y la Sierra Madre Oriental, en ella se encuentra una variedad de tipos de vegetación como selva baja caducifolia (Bursera morelensis, Cordia boissierii), matorral submontano (Fouquierria splendens, Ipomoea sp., Opuntia sp., Stenocereus queretaroensis, Prosopis laevigata) y bosque de pino-encino (Pinus michoacana, P. teocote,

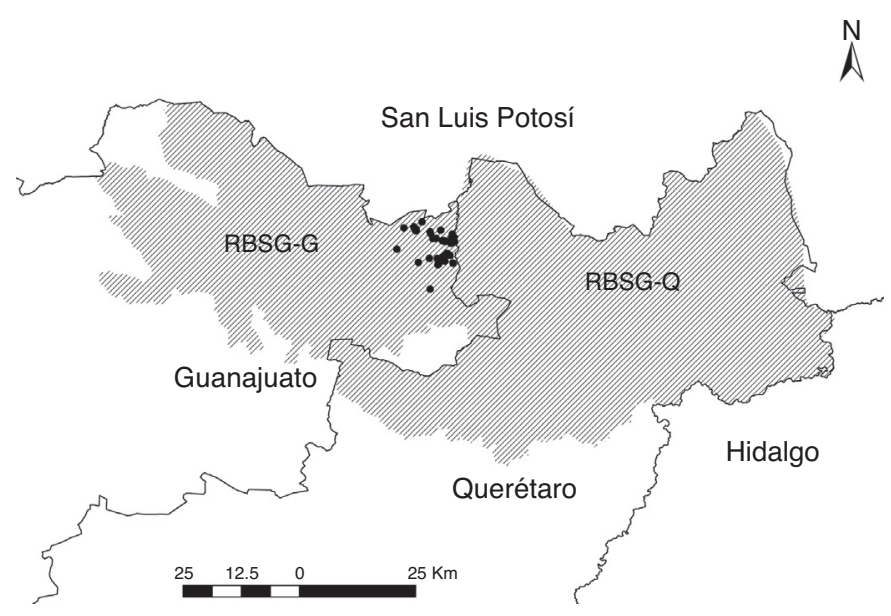

Figura 1. Ubicación geográfica de las Reservas de la Biosfera Sierra Gorda de Guanajuato (RBSG-G) y Querétaro (RBSG-Q). Los puntos negros indican la ubicación de los sitios de muestreo.

Juniperus flaccida, Quercus laurina, Q. affinis, Q. castanea, Q. crassifolia, Q. rugosa; Semarnat, 2007), intercalados entre sí a través de una accidentada topografía (Carranza, 2005). Recientemente el sitio se designó como un área natural protegida Reserva de la Biosfera Sierra Gorda de Guanajuato (Semarnat, 2007).

El trabajo de campo se realizó en 15 sitios localizados en la sección oriental de la Reserva de la Biosfera Sierra Gorda (fig. 1) y comprendió 6 visitas entre noviembre de 2011 y agosto de 2012, abarcando un total de 28 días con 191 h de trabajo de campo repartidos por estación del año. Se empleó el método de conteo por parcelas $(\mathrm{N}=8)$ con radio fijo dispuestas al azar en los principales tipos de vegetación, así como en cultivos y pastizales (Hutto, Pletschet y Hendricks, 1986; Ralph, Saber y Droege, 1995), en donde se registraron todas las especies de aves observadas y escuchadas durante 5 min dentro de una circunferencia con radio de $25 \mathrm{~m}$ y con una distancia mínima de $250 \mathrm{~m}$ entre cada parcela (Hutto et al., 1986). Los conteos se llevaron a cabo por la mañana entre las 06:30-11:30 h y por la tarde entre las 15:00-19:00 h; en pocas ocasiones los horarios fueron modificados por las condiciones ambientales de lluvia o neblina. Las observaciones se hicieron con binoculares $(8 \times 35$ y $10 \times 50)$. Además, se capturaron ejemplares mediante el uso de 6 redes ornitológicas $(2.5 \times 12 \mathrm{~m}$ y abertura de malla de $36 \mathrm{~mm}$ ), las cuales fueron colocadas a lo largo de cuerpos de agua, en los bordes de cultivos con remanentes de vegetación natural, en áreas de pastoreo y en los bordes de bosque y selva. Las redes se abrieron diariamente durante $4 \mathrm{~h}$ (07:00-11:00 h), si las condiciones climáticas y la presencia de personas o ganado lo permitían, sumando un total de $24 \mathrm{~h}$ red/día. La identificación de las especies se realizó con ayuda de guías especializadas (Dunn y Aldefer, 2006; Howell y Webb, 1995; Peterson y Chalif, 1989; Pyle, Howell y Yunick, 1987). Con el fin de estimar la exhaustividad del inventario se generó una curva de acumulación de especies en función del esfuerzo de muestreo, usando como unidad de esfuerzo el número de días (Chao, 2004). Se determinó que los estimadores de Jacknife de primer (Jack 1) y segundo orden (Jack 2) fueron los que mejor 


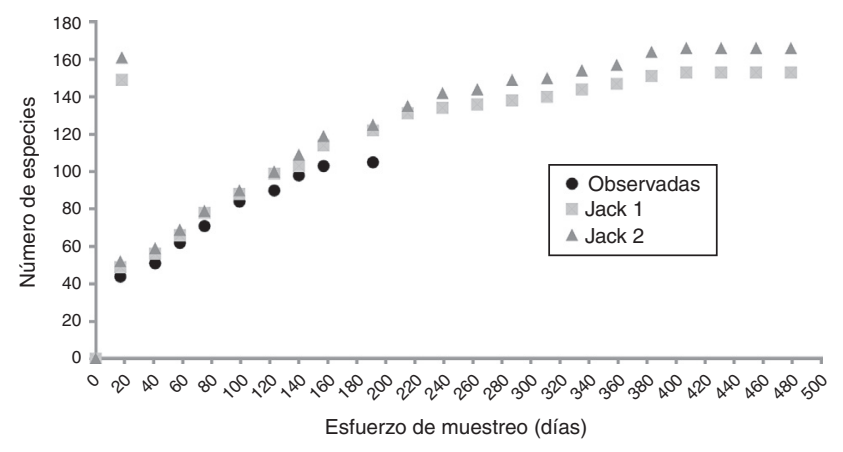

Figura 2. Curva de acumulación de especies de aves observadas y estimadas durante el periodo de estudio en la Reserva de la Biosfera Sierra Gorda de Guanajuato.

se ajustaron a los datos observados. El programa EstimateS 9.0 (Colwell, 2013) se utilizó para calcular el número de especies esperadas. La estacionalidad de las especies se determinó conforme a las categorías establecidas por Howell y Webb (1995) y complementadas con las observaciones realizadas en campo. Se aplicaron los criterios sugeridos por Sánchez-González (2013) para la publicación de nuevos registros, quien explica que un nuevo registro debe ser considerado como auténtico si los taxones se registran por primera vez en la entidad política o se registran en una región biótica distinta. También fueron utilizados como referencia de la novedad de la información los mapas de distribución potencial elaborados por Navarro-Sigüenza y Peterson (2007) de las especies consideradas en este trabajo (Conabio, 2008; www.conabio.gob.mx/informacion/gis/).

\section{Resultados}

Se registraron un total de 105 especies de aves, de las cuales 32 son migratorias y 73 son residentes (Anexo). Los estimadores utilizados en la curva de acumulación de especies no mostraron un valor cercano a lo observado (fig. 2). El modelo de acumulación de especies indica que se observaron aproximadamente el $68.6 \%$ (Jack 1) y el $63.2 \%$ (Jack 2) de la avifauna esperada en el área de estudio, con base en los métodos empleados. Lo anterior sugiere que el esfuerzo de muestreo invertido no fue el suficiente aun para detectar la totalidad de las especies potenciales para el área de estudio. A continuación se documenta la presencia de 7 especies que representan registros notables para Guanajuato; de las cuales 5 especies amplían su distribución conocida (Trogon mexicanus, Ara militaris, Cyanocorax yncas, Euphonia elegantissima y Spinus notatus) y 2 no estaban registradas para la entidad (Buteogallus anthracinus y Myiozetetes similis).

\section{Buteogallus anthracinus (aguililla negra-menor)}

Se observaron ocasionalmente 1-2 individuos sobrevolando los alrededores de la localidad de El Platanal, del río Santa María y en cañadas adyacentes con arroyos temporales entre marzo y agosto de 2012. Las marcas distintivas observadas en B. anthracinus fueron la coloración negra, alas anchas, una banda ancha terminal blanca en la cola y las patas largas amarillas; en vuelo resalta una franja blanca en la base de las rémiges primarias (Howell y Webb, 1995). Es una especie residente de la parte oriental de México (Howell y Webb, 1995). Especie no documentada en esta región, sin embargo, existen registros previos en la Sierra Gorda en Querétaro (González, 2001, 2008). Esta especie está enlistada bajo protección especial en México en la NOM-059 (Semarnat, 2010).

\section{Trogon mexicanus (trogón mexicano)}

Se observaron con regularidad 1-2 individuos perchando y vocalizando en un bosque de pino-encino cercano a la localidad de Puerto de Buenavista, de noviembre 2011 a agosto 2012. Las marcas distintivas observadas en T. mexicanus fueron la coloración verde brillante, vientre rojo y 3 franjas blancas en la cola negra (Howell y Webb, 1995). Esta especie ha sido registrada previamente en la Sierra Gorda, en bosques mesófilos y de pino-encino en la parte noroeste de Querétaro (González, 2001, 2008), pero no documentada para la parte de Guanajuato. También ha sido registrada en bosques mesófilos, de coníferas y húmedos de la Huasteca Potosina (Sánchez-González y GarcíaTrejo, 2010) y en el noreste de Hidalgo (Howell y Webb, 1995; Martínez-Morales, 2001). Es probable que habite en las partes más inaccesibles en los bosques de la Sierra Madre Oriental. Howell y Webb (1995) documentan esta especie como residente en el interior del país del sur de Chihuahua y Tamaulipas hasta Chiapas. Este registro amplía el área de distribución conocida de la especie en la Sierra Madre Oriental en aproximadamente $40 \mathrm{~km}$ al oeste (Howell y Webb, 1995).

\section{Ara militaris (guacamaya verde)}

Se observaron grupos conformados por 10-22 individuos durante noviembre de 2011 y agosto de 2012, sobrevolando, perchando y en busca de zonas de forrajeo en diferentes sitios del municipio de Xichú (Puerto de Buenavista, El Roblar, El Tepozán, Las Palomas, El Gato, El Platanal, El Pinalito). Las marcas distintivas observadas en A. militaris fueron la coloración verde brillante, las plumas primarias y de la rabadilla azules (Howell y Webb, 1995). Estos individuos probablemente provienen del Sótano del Barro en Querétaro. A la fecha no se han registrado sitios de anidación y de alimentación en ninguna región de Guanajuato. La distribución conocida de la especie se amplía en la sierra Madre Oriental en aproximadamente $20 \mathrm{~km}$ al oeste, pues ha sido registrada previamente en bosques de pino-encino en la parte noroeste de Querétaro (Gaucín, 2000; González, 2001, 2008), agregando una nueva localidad de presencia a este taxón de gran interés para la conservación.

Myiozetetes similis (luis gregario)

Dos individuos fueron vistos y escuchados regularmente perchando en árboles de Bursera y alimentándose de insectos, entre marzo y agosto de 2012 a $780 \mathrm{~m}$ snm a las orillas del río Santa María y cercanos a la localidad de El Platanal. Otros 2 individuos fueron observados en el camino entre las localidades de Puerto de Buenavista y El Platanal a $830 \mathrm{~m}$ snm. Las marcas distintivas 
observadas en $M$. similis fueron el pico pequeño de color negro, coloración amarilla en el vientre y parche naranja rojizo en la corona (Howell y Webb, 1995). Esta especie ha sido registrada en bosques mesófilos, de coníferas y húmedos de la Huasteca Potosina (Sánchez-González y García-Trejo, 2010). Esta es una especie residente, que a pesar de ser común a lo largo de las costas y tierras bajas, frecuentemente se observa cerca de poblados, muy raramente es registrada en sitios al interior del país (Howell y Webb, 1995) y no ha sido previamente registrada en esta región; sin embargo, existen registros cercanos previos en la Sierra Gorda en Querétaro (González, 2001, 2008).

\section{Cyanocorax yncas (chara verde)}

Grupos de 2-4 individuos fueron observados con regularidad en cañadas de selva baja perturbada asociada con vegetación riparia en el camino entre las localidades de Puerto de Buenavista y El Platanal, a $830 \mathrm{~m}$ snm, entre noviembre de 2011 a agosto de 2012. Las marcas distintivas observadas en $C$. yncas fueron la coloración verde, el parche negro en la garganta y corona azul (Howell y Webb, 1995). Es residente en la vertiente del golfo de México, desde Tamaulipas hasta Chiapas, incluyendo la península de Yucatán; y en la vertiente del Pacífico, desde el sur de Nayarit a Oaxaca (Howell y Webb, 1995). Este registro extiende el área de distribución conocida de la especie en la Sierra Madre Oriental en aproximadamente $70 \mathrm{~km}$ al oeste de sus localidades de presencia en la Sierra Gorda en Querétaro (González, 2001, 2008) y de la Huasteca Potosina (SánchezGonzález y García-Trejo, 2010).

\section{Euphonia elegantissima (eufonia capucha-azul)}

Observada con regularidad forrajeando durante noviembre de 2011 a marzo de 2012 en bosque de pino y en los bordes de bosque de los diferentes caminos a $1,950 \mathrm{~m}$ snm. Las marcas distintivas observadas en E. elegantissima: en el macho fueron el vientre canela, partes posteriores del cuello azul; mientras que en la hembra, coloración verde-oliváceo, garganta canela y corona azul (Howell y Webb, 1995). Residente en ambas vertientes desde Sonora y Tamaulipas, el interior del centro de México hasta Chiapas (Howell y Webb, 1995). Este registro amplía el área de distribución conocida en la Sierra Madre Oriental en aproximadamente $80 \mathrm{~km}$ al oeste, pues ha sido registrada previamente en bosques de pino-encino en la parte noroeste de Querétaro (Gaucín, 2000; González, 2001, 2008).

\section{Spinus notatus (jilguero encapuchado)}

Este jilguero se observó con regularidad (2-8 individuos) en espacios abiertos en los alrededores de las localidades de Puerto de Buenavista, El Roblar y El Tepozán entre noviembre de 2011 a marzo de 2012, en zonas de pastoreo y cultivos principalmente, y en zonas con vegetación herbácea con remanentes de bosque de pino-encino. Las marcas distintivas observadas en $S$. notata fueron el pecho y cabeza negra, partes inferiores amarillas y parches de color amarillo en las alas y cola (Howell y Webb, 1995). Residente del sur de Sonora y sur de Tamaulipas hasta
Chiapas (Howell y Webb, 1995). Este registro amplía su área de distribución conocida en la Sierra Madre Oriental por $40 \mathrm{~km}$ al oeste, pues ha sido registrada previamente en bosques de pino-encino en la parte noroeste de Querétaro (González, 2001, 2008).

\section{Discusión}

La Reserva de la Biosfera de la Sierra Gorda en Guanajuato es un área que ha sido muy poco explorada de forma sistemática, por lo que el listado de aves que se presenta en el documento de registro oficial de su estatus como reserva de la biosfera carece de un sustento completo de inventarios ornitológicos (Conanp, 2005), como puede demostrarse por los registros nuevos presentados aquí para el estado. De las áreas que cubre la reserva, nuestros recorridos de campo en la zona de Xichú nos muestran que es una de las que presenta mejor estado de conservación, a pesar de que existe cierta actividad ganadera, forestal y minera en la zona. Esta zona presenta diversos tipos de vegetación como bosques de pino y encino, pastizales y cultivos; mientras que hacia el oeste se ubican algunas zonas de matorral y la zona de bosque tropical seco con barrancas bien conservadas y está alejada de la zona con centros urbanos. Comparada esta zona con la región adyacente del estado de Querétaro, la cual es más seca con matorral y solo existe vegetación riparia y manchones de bosque tropical en las barrancas y zonas cercanas a cuerpos de agua. Esto quizás sea un factor importante para la presencia de algunas especies como las aquí documentadas. Las posibles razones de la ausencia previa de registros en el área de las 7 especies que se analizaron, especialmente el caso de $A$. militaris, son, además de una probable expansión reciente de sus rutas de desplazamiento, que la especie estaba, pero no se había registrado en la zona o que sí se había documentado, pero esos registros no se han publicado (Gaucín, 2000). La adición de 7 especies de aves para el estado de Guanajuato y el registro de otras 98 especies sugieren la importancia de esta región a nivel estatal como un área importante de riqueza de especies de aves y como zona de contacto de aves de afinidad tropical distribuidas en el este de México con las de ambientes secos del Altiplano Mexicano, así como por la presencia de especies indicadoras de buen estado de conservación de los hábitats de bosque, como T. mexicanus, A. militaris y Xiphorhynchus flavigaster, por mencionar algunas. Sin embargo, es necesario continuar con los esfuerzos de investigación que permitan completar el conocimiento de la avifauna de la Sierra Gorda de Guanajuato y así poder evaluar el estado de conservación real de la comunidad de aves y de las poblaciones que la componen.

\section{Agradecimientos}

A C. Vázquez, L. del Villar y a M. Pérez por su ayuda durante el trabajo de campo. A T. Olvera y a la familia VelázquezGonzález su apoyo y hospedaje en campo. Así como a 2 revisores anónimos sus comentarios, que mejoraron la calidad de este trabajo. 
Anexo. Listado de especies de aves (de acuerdo con AOU, 2015) registradas en los diferentes sitios de estudio de la Reserva de la Biosfera Sierra Gorda de Guanajuato, indicando el estatus migratorio (sensu Howell y Webb, 1995), la categoría de riesgo (sensu Semarnat, 2010) y la categoría de endemismo (sensu González-García y Gómez de Silva, 2003; Navarro-Sigüenza y Benítez, 1993)

\begin{tabular}{lcc}
\hline Orden & & Estatus migratorio \\
\hline Familia & Especie &
\end{tabular}

\section{Anseriformes \\ Anatidae \\ Galliformes \\ Cracidae \\ Pelecaniformes \\ Ardeidae \\ Egretta thula \\ Accipitriformes \\ Cathartidae \\ Accipitridae \\ Charadriiformes \\ Scolopacidae \\ Columbiformes \\ Columbidae}

Cuculiformes

Cuculidae

Strigiformes

Strigidae

Caprimulgiformes Caprimulgidae

Apodiformes

Trochilidae

Trogoniformes

Trogonidae

Coraciformes

Alcedinidae

Piciformes

Picidae
Anas discors

Ortalis vetula

Ardea alba

Bubulcus ibis

Coragyps atratus

Cathartes aura

Buteogallus anthracinus Buteo jamaicensis

Actitis macularius

Patagioenas fasciata

Zenaida asiatica

Zenaida macroura

Columbina inca

Columbina passerina

Geococcyx californianus

Glaucidium brasilianum

Chordeiles acutipennis

Residente

Archilochus colubris

Cynanthus latirostris

Hylocharis leucotis

Trogon mexicanus

Chloroceryle americana

Melanerpes formicivorus Sphyrapicus varius

Residente

Migratoria

Residente

Residente

Residente $^{\mathrm{a}}$

Residente

Migratoria

Residente

Residente

Residente

Residente

Residente

Residente

Residente

Residente

Residente

Residente Migratoria
Colibri thalassinus

Migratoria

Residente

Residente

Residente

Migratoria
Orden

Estatus migratorio

\begin{tabular}{l}
\hline Familia \\
\hline \\
Falconiformes \\
Falconidae \\
Psittaciformes \\
Psittacidae \\
Passeriformes \\
Furnariidae \\
Tyrannidae
\end{tabular}

Residente
Vireonidae

Corvidae

Hirundinidae

Paridae

Aegithalidae

Sittidae

Certhiidae

Troglodytidae

Polioptilidae

Regulidae

Turdidae

Mimidae

Ptilogonatidae

Parulidae
Falco sparverius

Falco mexicanus

Ara militaris

Xiphorhynchus flavigaster

Contopus pertinax

Empidonax hammondi

Sayornis nigricans

Sayornis saya

Pyrocephalus rubinus

Myiarchus cinerascens

Myiozetetes similis

Tyrannus vociferans

Vireo huttoni

Vireo gilvus

Cyanocorax yncas

Cyanocitta stelleri

Aphelocoma wollweberi

Corvus corax

Stelgidopteryx serripennis

Hirundo rustica

Baeolophus wollbeberi

Psaltriparus minimus

Sitta carolinensis

Certhia americana

Salpinctes obsoletus

Catherpes mexicanus

Troglodytes aedon

Thryomanes bewickii

Polioptila caerulea

Regulus calendula

Sialia mexicana

Myiadestes occidentalis

Turdus grayi

Turdus migratorius

Melanotis caerulescens

Toxostoma curvirostre

Mimus polyglottos

Ptilogonys cinereus

Mniotilta varia
Residente

Residente

Residente

Residente Migratoria $^{b}$

Residente $^{c}$

Residente

Residente

Migratoria

Residente

Residente

Residente

Migratoria

Residente

Residente

Residente

Migratoria

Residente

Residente

Residente

Residente

Migratoria

Migratoria

Residente

Residente

Residente

Residente

Residente

Residente

Migratoria

Residente

Residente

Migratoria

Residente

Residente

Residente

Migratoria

Residente $^{\mathrm{d}}$

Residente

Residente

Residente

Migratoria 


\begin{tabular}{|c|c|c|}
\hline \multicolumn{2}{|l|}{ Orden } & \multirow[t]{2}{*}{ Estatus migratorio } \\
\hline Familia & Especie & \\
\hline & Oreothlypis ruficapilla & Migratoria \\
\hline & Geothlypis tolmiei & Migratoria \\
\hline & Setophaga pitiayumi & Migratoria \\
\hline & Setophaga petechia & Migratoria \\
\hline & Setophaga coronata & Migratoria \\
\hline & Setophaga nigrescens & Migratoria \\
\hline & Setophaga occidentalis & Migratoria \\
\hline & Setophaga townsendi & Migratoria \\
\hline & Basileuterus rufifrons & Residente \\
\hline & Cardellina pusilla & Migratoria \\
\hline & Myioborus pictus & Residente \\
\hline & Myioborus miniatus & Residente \\
\hline \multicolumn{3}{|c|}{ Emberizidae } \\
\hline & Pipilo maculatus & Residente \\
\hline & Melozone fusca & Residente \\
\hline & Spizella passerina & Residente \\
\hline & Pooecetes gramineus & Migratoria \\
\hline & Chondestes grammacus & Migratoria \\
\hline & Melospiza lincolnii & Migratoria \\
\hline & Junco phaeonotus & Residente \\
\hline \multicolumn{3}{|c|}{ Cardinalidae } \\
\hline & Piranga flava & Residente \\
\hline & Piranga rubra & Migratoria \\
\hline & Piranga ludoviciana & Migratoria \\
\hline & Pheucticus melanocephalus & Residente \\
\hline & Passerina caerulea & Residente \\
\hline & Passerina versicolor & Residente \\
\hline \multicolumn{3}{|c|}{ Icteridae } \\
\hline & Molothrus aeneus & Residente \\
\hline & Molothrus ater & Residente \\
\hline & Icterus wagleri & Residente \\
\hline & Icterus spurius & Migratoria \\
\hline & Icterus cucullatus & Migratoria \\
\hline & Icterus bullockii & Residente \\
\hline & Icterus graduacauda & Residente \\
\hline \multicolumn{3}{|c|}{ Fringillidae } \\
\hline & Euphonia elegantissima & Residente \\
\hline & Haemorhous mexicanus & Residente \\
\hline & Spinus notatus & Residente \\
\hline & Spinus psaltria & Residente \\
\hline & Coccothraustes abeillei & Residente \\
\hline
\end{tabular}

a $=$ protección especial; ${ }^{b}=$ amenazada $;{ }^{c}=$ peligro de extinción; ${ }^{\mathrm{d}}=$ endémica.

\section{Referencias}

AOU (American Ornithologists' Union). (2015). AOU checklist of North and Middle American birds. (Seventh edition and Supplements). Recuperado el 28 de mayo de 2015 de: http://checklist.aou.org/

Bolaños, R., Sánchez-Cordero, V., Gurrola-Hidalgo, M. A., Iglesias, J. A., Magaña-Cota, G. E. y Botello, F. J. (2012). Primer registro de la cojolita (Penelope purpurascens) en el estado de Guanajuato, México. Acta Zoológica Mexicana (nueva serie), 26, 237-241.

Carranza, E. (2005). Conocimiento actual de la flora y la diversidad vegetal del estado de Guanajuato, México. En J. Rzedowsky y G. Calderón de Rzedowsky (Eds.), Flora del Bajío y de regiones adyacentes. Fascículo complementario (21) (p. 17). México, D.F: Instituto de Ecología, A.C.

Chao, A. (2004). Species richness estimation. En N. Balakrishnan, C. B. Read, y B. Vidakovic (Eds.), Encyclopedia of statistical sciences (pp. 1-23). New York: Wiley.

Chapa-Vargas, L., Salgado-Ortiz, J. y Monzalvo-Santos, K. (2011). Designing actions for bird conservation at the Yuriria Lagoon, it's associated floodplain and terrestrial habitats in Michoacán, Mexico. En M. A. Álvarez (Ed.), Floodplains: physical geography, ecology and societal interactions (pp. 29-54). New York: Nova Science Publishers.

Colwell, R.K. (2013). EstimateS: Statistical estimation of species richness and shared species from samples. Version 9.0. User's guide and application. Recuperado el 1 de mayo de 2015 de: http://viceroy.eeb. uconn.edu/estimates.

Conabio (Comisión Nacional para el Conocimiento y Uso de la Biodiversidad). (2008). División política y estatal escala 1:250,000. Recuperado el 14 de septiembre de 2014 de: http://www.conabio.gob.mx/ informacion/metadata/gis/.

Conanp (Comisión Nacional de Áreas Naturales Protegidas). (2005). Estudio previo justificativo para el establecimiento del Área Natural Protegida Reserva de la Biosfera Sierra Gorda de Guanajuato. México, D.F.: ConanpSemarnat.

Cruz, A. (1986). Contribución al conocimiento de la avifauna de los alrededores de la laguna de Yuriria, del Bajío del estado de Guanajuato, México. Tesis. Facultad de Ciencias, Universidad Nacional Autónoma de México.

Donagho, W. R. (1965). The starling in Guanajuato, Mexico. Condor, 67, 447.

Dunn, J. L. y Aldefer, J. (2006). Field guide to the birds of North America (4th Ed.). Washington, D.C: National Geographic Society.

Esparza-Claudio, J. J. (2012). Cambio en el uso de suelo y degradación ambiental. En Conabio (Ed.), La biodiversidad en Guanajuato: estudio de estado (pp. 327-331). Guanajuato: Conabio-Instituto de Ecología del Estado de Guanajuato.

Estrada, A. (1995). Estudio preliminar de la avifauna de la sierra de Santa Rosa, Guanajuato, México. Tesis. Facultad de Estudios Superiores Iztacala, Universidad Nacional Autónoma de México.

Gaucín, N. (2000). Biología de la conservación de la guacamaya verde (Ara militaris) en el Sótano del Barro, Querétaro. Universidad Autónoma de Querétaro. Facultad de Ciencias Naturales. México, D.F.: Informe final SNIB-Conabio proyecto No. L204.

Gómez-Rodríguez, J. (2011). Comunidades de aves en humedales palustres y riberinos de vocación agrícola en Pénjamo, Guanajuato y La Piedad, Michoacán. Tesis. Universidad Michoacana de San Nicolás de Hidalgo.

González, A. V. (2012). La riqueza avifaunística como elemento para la conservación del bosque templado de la sierra de Santa Rosa. En Conabio (Ed.), $\mathrm{La}$ biodiversidad en Guanajuato: estudio de estado (pp. 257-262). Guanajuato: Conabio-Instituto de Ecología del Estado de Guanajuato.

González, C. (2001). Avifauna de la Reserva de la Biosfera Sierra Gorda, Querétaro, México. Tesis. Facultad de Estudios Superiores Iztacala, Universidad Nacional Autónoma de México.

González, C. (2008). Composición avifaunística de la Reserva de la Biosfera Sierra Gorda, Querétaro, México. Tesis de maestría. Instituto de Biología, Universidad Nacional Autónoma de México.

González-García, F. y Gómez de Silva, H. (2003). Especies endémicas: riqueza, patrones de distribución y retos para su conservación. En H. Gómez de Silva y A. Oliveras de Ita (Eds.), Conservación de aves. Experiencias en México (pp. 150-194). México, D.F: National Fish and Wildlife FoundationConabio.

Gurrola-Hidalgo, M. A., Escalante, P., López, A. S. y Sanabria, F. T. (2012). Aves. En Conabio (Ed.), La biodiversidad en Guanajuato: estudio de estado (pp. 244-254). Guanajuato: Conabio-Instituto de Ecología del Estado de Guanajuato

Howell, S. N. G. y Webb, S. (1995). A guide to the birds of Mexico and Northern Central America. New York: Oxford University Press.

Hutto, R. L., Pletschet, S. M. y Hendricks, P. (1986). A fixed-radius point count method for non-breeding and breeding season use. Auk, 103, 593-602.

Labarthe, V. (2004). Las aves de la sierra de Santa Rosa, Guanajuato. Guanajuato: Fundación Ecológica de Guanajuato, A.C., Instituto Estatal de Ecología del Estado de Guanajuato.

Martínez-Morales, M. (2001). Forest fragmentation effects on bird communities of tropical montane cloud forests in Eastern Mexico. Tesis doctoral. University of Cambridge.

Moore, R. T. (1939). A new race of Cynanthus latirostris from Guanajuato. Proceedings of Biological Society of Washington, 53, 23-29. 
Moya, L.E. (2012). Diversidad de los vertebrados terrestres en la sub-cuenca Esperanza-Soledad-Santa Ana, del municipio de Guanajuato, Guanajuato. Tesis. Facultad de Estudios Superiores Iztacala, Universidad Nacional Autónoma de México.

Navarro-Sigüenza, A. G. y Benítez, H. (1993). Patrones de riqueza y endemismo de las aves. Ciencias, 7, 45-54

Navarro-Sigüenza, A. G., Garza-Torres, H. A., López de Aquino, S., RojasSoto, O. y Sánchez-González, L. A. (2004). Patrones biogeográficos de la avifauna. En I. Luna, J. J. Morrone, y D. Espinosa (Eds.), Biodiversidad de la sierra Madre Oriental (pp. 439-467). México, D.F: Las Presas de Ciencias.

Navarro-Sigüenza, A.G. y Peterson, A.T. (2007). Mapas de las aves de México. Distribución potencial. Extraído del proyecto CE015. Museo de Zoología, Facultad de Ciencias, UNAM-University of Kansas, Museum of Natural History. Financiado por la Conabio. México. Recuperado el 24 de abril de 2014 de: http://www.conabio.gob.mx/informacion/gis.

Navarro-Sigüenza, A. G., Rebón-Gallardo, M. F., Gordillo-Martínez, A., Peterson, A. T., Berlanga, H. y Sánchez-González, L. A. (2014). Biodiversidad de aves en México. Revista Mexicana de Biodiversidad, 85(Supl.), S476-S495.

Peterson, R. T. y Chalif, E. L. (1989). Aves de México: guía de campo. México, D.F: Editorial Diana.

Pyle, P., Howell, S. y Yunick, R. (1987). Identification guide to North American passerines: a compendium of information on identifying, aging, and sexing passerines in the hand. Bolinas: Islate Creek Press.

Ralph, C. J., Saber, J. R. y Droege, S. (1995). Monitoring bird populations by point counts. General Technical Report PSW-GTR-149. Albany: USDA Forest Service, Pacific Southwest Research Station.

Rodríguez-Yáñez, C., Villalón, R. y Navarro-Sigüenza, A. G. (1994). Bibliografía de las aves de México (1825-1992). Publicación Especial del Museo de Zoología, 4, 1-146.

Rojas-Soto, O. y Oliveras de Ita, A. (2005). Los inventarios avifaunísticos: reflexiones sobre su desarrollo en el Neotrópico. Ornitología Neotropical, 16, $441-445$.
Sánchez-Cordero, V. (2007). Inventario de la diversidad de mamíferos, anfibios, reptiles y aves de cuatro áreas naturales protegidas al sur del estado de Guanajuato. México, D.F: Proyecto Conabio.

Sánchez-González, L. A. (2013). Cuando un «nuevo registro» es realmente un nuevo registro: consideraciones para su publicación. Huitzil, 14, 17-21.

Sánchez-González, L. A. y García-Trejo, E. (2010). San Luis Potosí. En R. Ortiz-Pulido, A. G. Navarro-Sigüienza, H. Gómez de Silva y A. T. Peterson (Eds.), Avifaunas estatales de México (pp. 199-242). Pachuca: CIPAMEX.

Semarnat (Secretaría de Medio Ambiente y Recursos Naturales). (2007). Decreto por el que se declara área natural protegida, con el carácter de reserva de la biosfera, la zona conocida como Sierra Gorda de Guanajuato localizada en los municipios de Atarjea, San Luis de la Paz, Santa Catarina, Victoria y Xichú, en el estado de Guanajuato. Diario Oficial de la Federación. 2 de febrero de 2007, Primera Sección. México.

Semarnat (Secretaría de Medio Ambiente y Recursos Naturales). (2010). Norma Oficial Mexicana NOM-059-SEMARNAT-2010, Protección ambientalEspecies nativas de México de flora y fauna silvestres-Categorías de riesgo y especificaciones para su inclusión, exclusión o cambio-Lista de especies en riesgo. Diario Oficial de la Federación. 30 de diciembre del 2010, Segunda Sección. México.

Uriarte-Garzón, P. (2012). Monitoreo de aves acuáticas dentro del Área Natural Protegida Cerro de Arandas. En Conabio (Ed.), La biodiversidad en Guanajuato: estudio de estado (pp. 255-256). Guanajuato: Conabio-Instituto de Ecología del Estado de Guanajuato.

Uriarte-Garzón, P. y Lozoya, G. E. (2009). Manual del inventario de la fauna del Área Natural Protegida Cerro de Arandas. Irapuato: Parque Ecológico de Irapuato, A.C.

Zuria, I. (2003). Birds and field margins in an agricultural landscape of Guanajuato, Mexico. Tesis doctoral. University of Maryland.

Zuria, I. y Gates, J. E. (2013). Community composition, species richness, and abundance of birds in field margins of central Mexico: local, and landscapescale effects. Agroforestry Systems, 87, 377-393. 